\title{
Tool path planning in milling of repaired blades of blisks
}

\author{
Yazhou Feng ${ }^{1,}$, Junxue Ren ${ }^{1, b}$ and Yingying $\mathrm{Xu}^{1, \mathrm{c}}$ \\ ${ }^{1}$ School of Mechanical Engineering, Northwestern Polytechnical University, Xi'an 710072, China \\ aasian5921@163.com, ${ }^{\text {brjx1968@nwpu.edu.cn, }{ }^{c} 1016529685 @ q q . c o m}$
}

Keywords: Blisks, Repaired blade, Repairing and machining, Tool path planning; Machining allowance

Abstract. A tool path planning approach is proposed in this paper to machine repaired blades of blisks in rough and finish milling. Measured allowances on the blank of the repaired blade are first analyzed with the measured point data. With respect to the non-uniform allowance on the blank, the cutting region for rough milling is determined and tool path planning is conducted based on the proposed iso-stepover method. The cutting region for finish milling is then determined to guarantee that the cutting region can be smoothly connected to the non-cutting region. Finally, a tool path modification method is proposed when the transition region is included.

\section{Introduction}

Blisks integrate blades and hubs together instead of traditional usages of the dovetail attachment. This integrated structure reduces the engine's weight and part count while improves its efficiency and reliability. However, the flexibility in production and maintenance of blisks is correspondingly reduced. On the one hand, the damaged blades caused by poor tool path or operator's carelessness in manufacturing will lead the whole blisk to be abandoned. On the other hand, some blade(s) accidentally unqualified or damaged in service will also lead the whole blisk to be retired in advance. These include that (a) the fatigue fracture, edge fracture, and reduced reliability caused by defects of cracks, corrosion, and wear, and (b) bird strike and inhalation of objects [1,2,3]. Due to the expensive manufacturing costs and long production cycle of blisks, the blade(s) damage will increase renewing costs and delay the service of new blisks dramatically. Therefore, the research on repairing and machining technique for damaged blades of blisks is beneficial to reducing the unqualified rate, manufacturing costs, and renewing cycle, which will promote the application of blisks in the new aero-engine.

The affected region of damaged blades on blisks is usually cut off and welded with a new part with the assistant of linear friction welding or laser cladding technology. However, location and shape accuracy of the new added part cannot satisfy the designed requirement of the blisks. The final accuracy and surface roughness still resort to the computer numerical control (CNC) machining. In this paper, the damaged region of the repaired blades is first analyzed to determine the machining region and distributions of machining allowance. Then tool trajectories and orientations are planned to machine the repaired blades.

\section{Machining Allowance on the Repaired Blades}

The repaired region on the blade is measured with a three-coordinate measuring machine. Then the measured points are matched with the designed model to construct the geometric model of the repaired blades. To analyze the machining allowance, the constructed model is compared with the designed model point by point. The distance between a point on the designed model and its corresponding point on the constructed model is adopted to present the machining allowance at this point. This distance is calculated as follows.

Step 1 Extract iso-parametric curves on the designed model along the direction from the blade root to blade tip, while extract sample points on each iso-parametric curve with a constant curve length. Denote one of these sample points as point $Q_{i}$. 
Step 2 Calculate the partial derivatives $r_{u}$ and $r_{v}$ at an arbitrary point $P(u, v)$ on the constructed model.

Step 3 Calculate the directional vector $S$ from point $P(u, v)$ to point $Q_{i}$ and decompose it into two directions $r_{u}$ and $r_{v}$ as $S=A r_{u}+B r_{v}$.

Step 4 Calculate the dot product of vector $S$ and partial derivatives $r_{u}$ and $r_{v}$ as

$$
\begin{aligned}
& S r_{u}=A r_{u}^{2}+B r_{v} r_{u} \\
& S r_{v}=A r_{u} r_{v}+B r_{v}^{2}
\end{aligned}
$$

Step 5 Solve the coefficients $A$ and $B$ and check whether Eq. 3 is satisfied with a given tolerance $\varepsilon$. If they are not satisfied, search for the next point; otherwise, the current point $P(u, v)$ is the nearest point to point $Q_{i} \cdot\left|S r_{u}\right| \leq \varepsilon$,

$$
\left|S r_{u}\right| \leq \varepsilon, \quad\left|S r_{v}\right| \leq \varepsilon
$$

Step 6 Conduct step 2 to step 5 for all sample points on the designed model and calculate the distance of each pair of points on two models. The calculated distances present the distribution of machining allowance on the repaired blade.

\section{Machining Region on the Repaired Blades}

Due to the irregular distribution of machining allowance, the repaired blades are machined with a rough milling and a finish milling. The rough milling removes most of the machining allowance on the repaired blades, while the finish milling machines the repaired blades to their designed model and guarantees that the repaired region can be smoothly connected to the norm region. So a repaired blade is divided into three regions: norm region $S_{1}$, transitional region $S_{2}$, and repaired region $S_{3}$. Here, transitional region $S_{2}$ is a special region with a given width to guarantee the smooth connection of norm region $S_{1}$ and repaired region $S_{3}$.

\section{Rough Milling Region}

Since the rough milling is mainly used to remove most of the redundant material on the repaired blades, the rough milling region is limited in repaired region $S_{3}$, as shown in Fig. 1.

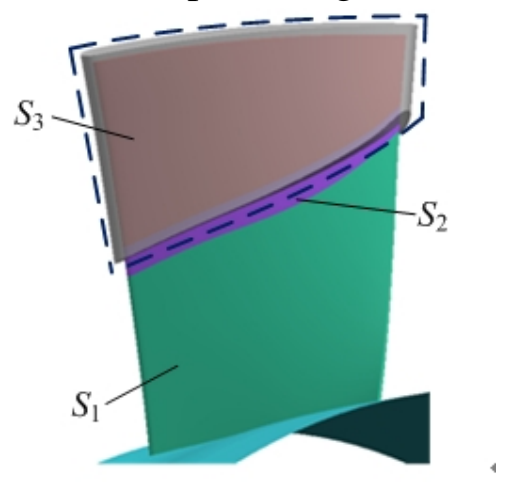

Fig. 1 Rough milling region

\section{Finish Milling Region}

A blade is usually machined with an iso-parametric curve method. To guarantee the smooth connection of norm region $S_{1}$ and repaired region $S_{3}$, transitional region $S_{2}$ should be completely covered with certain iso-parametric curves in finish milling. The exact iso-parametric curve which passes through the innermost point of the transitional region is selected to divide the repaired blade into two parts, as shown in Fig. 2. The part at which the repaired region is located is the machining 
region in finish milling.

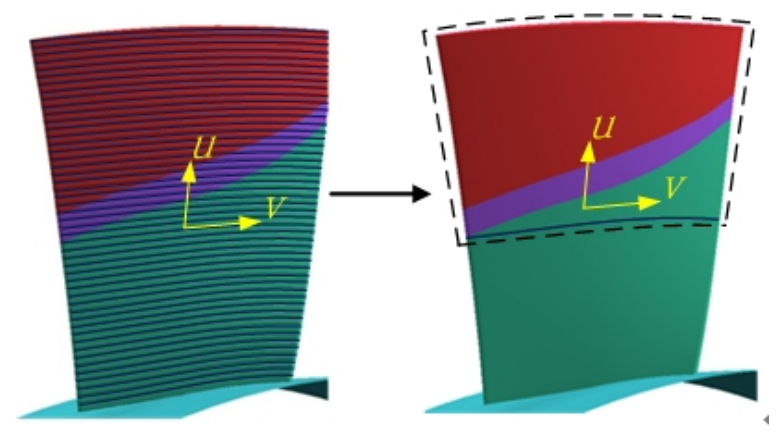

Fig. 2 Finish milling region

\section{Tool Path Planning}

\section{Tool Path Planning in Rough Milling}

Iso-parametric curve method and parallel section are two popular methods to plan tool trajectory in milling of blades. Since the length of leading edge is not the same as the trailing edge, an iso-stepover method is proposed in this paper to plan the tool trajectory in rough milling of repaired blades. Denote the constructed surface in the repaired region as surface $S(u, v)$. As shown in Fig. 3a, iso-parametric curves on this surface along the two parametric directions are denoted as $u_{i}(v)$ and $v_{j}(u)$, respectively. The iso-stepover method is introduced simply as follows.

Step 1 Calculate sample points $u_{i}\left(v_{j}\right)$ on curve $u_{i}(v)$ with a nearly constant stepover (or curve length), as shown in Fig. 3b.

Step 2 Interpolate B-spline curves $c_{j}(u)$ with sample points $u_{i}\left(v_{j}\right)$ line by line, as shown in Fig. $3 c$.

Step 3 Calculate sample points $c_{j}\left(u_{k}\right)$ on curve $c_{j}(u)$ with a nearly constant stepover with the same method as step 1, as shown in Fig. 3d.

Step 4 Project all sample points $c_{j}\left(u_{k}\right)$ onto surface $S(u, v)$.

Step 5 Interpolate new B-spline curves with the projected sample points.

After this process, the tool trajectory in rough milling is planned. Here, the projected points are used as the cutter location (CL) points .

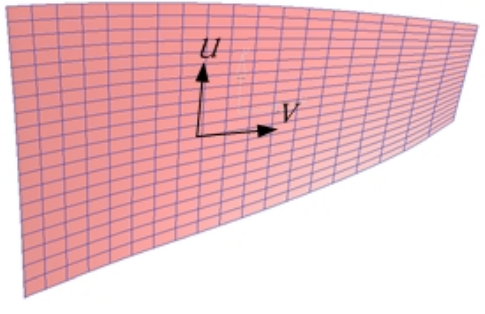

a. Iso-parametric curves on the surface

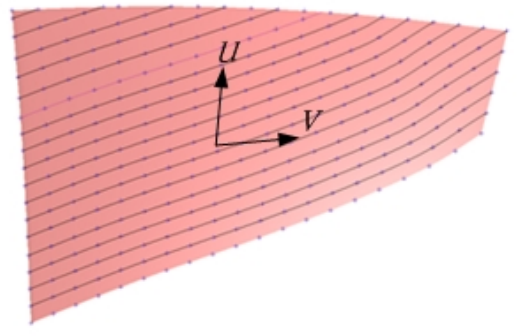

c. Interpolated B-spline curves

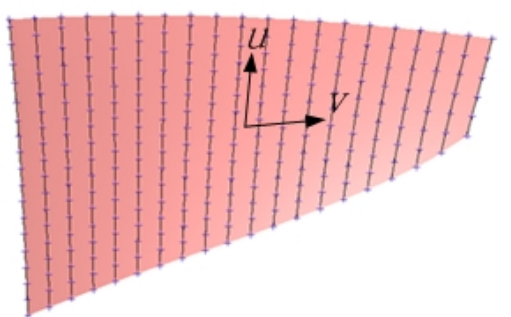

b. Sample points $u_{i}\left(v_{j}\right)$ on curve $u_{i}(v)$

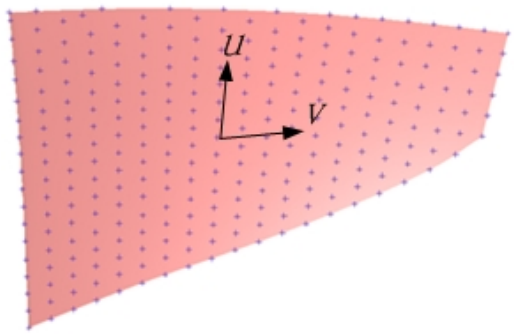

d. Sample points $c_{j}\left(u_{k}\right)$ on curve $c_{j}(u)$

Fig. 3 Tool trajectory planning in rough milling 


\section{Tool Path Planning in Finish Milling}

In this part, the region divisions are adjusted according to the iso-parameter curves of the blade surface. Denote the boundaries of the original transitional region as curves $c_{a}$ and $c_{b}$, while denote the iso-parametric curves related to these boundaries as curves $c_{1}, c_{2}$, and $c_{3}$, as shown in Fig. 4 . The new normal region $S_{2}^{\prime}$, new transitional region $S_{2}^{\prime}$, and completely repaired region $S_{3}^{\prime}$ are divided with respect to the iso-parametric curves. It is obvious that regions $S_{2}^{\prime}$ and $S_{3}^{\prime}$ are coincided with the finish milling region shown in Fig. 2.

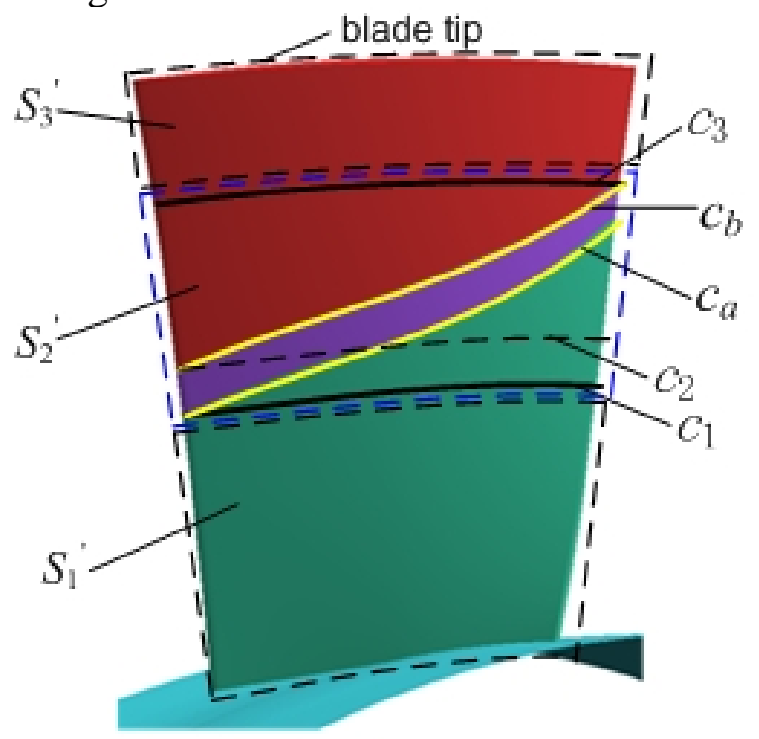

Fig. 4 New region divisions

Region $S_{3}^{\prime}$ is machined with the iso-parametric curve method, in which the CL points are calculated with the method introduced in rough milling, as shown in Fig. 5. Since the rough milling process leaves a constant allowance, the machining allowance in this region is so-called constant.

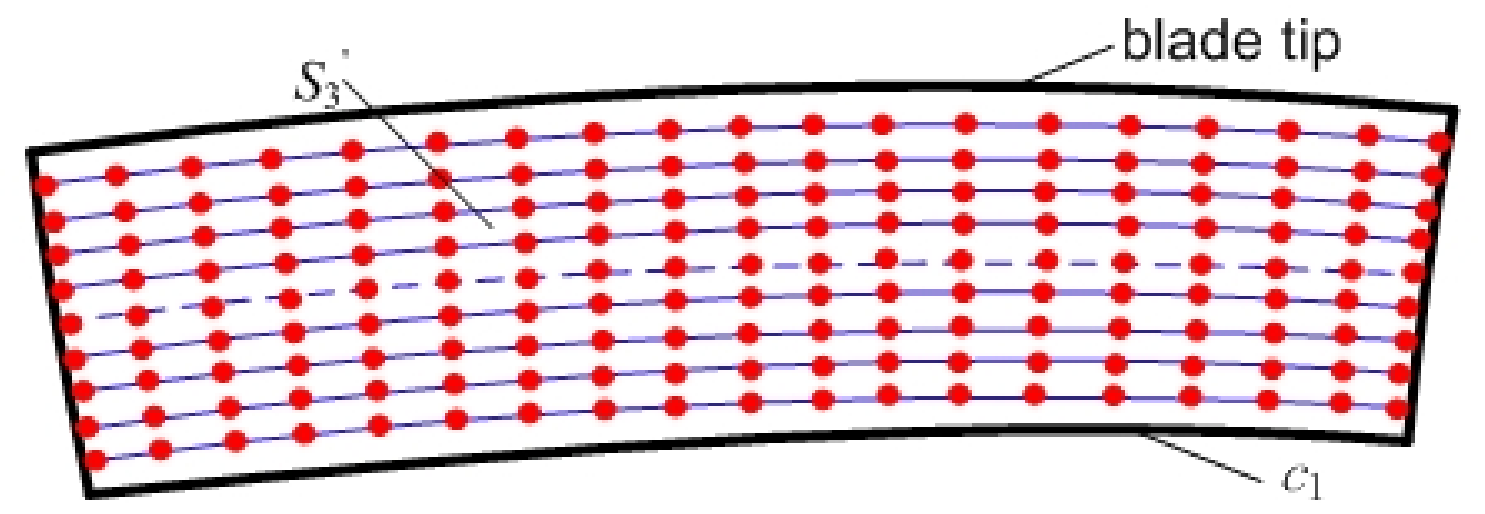

Fig. 5 Tool trajectories in finish milling of the completely repaired region $S_{3}^{\prime}$

The non-uniformly distributed allowance is adopted to plan tool path in the new transitional region $S_{2}^{\prime}$ to guarantee the uniformity on the machined surface. Suppose the iso-parameter curves shown in Fig. 4 are distributed along the $u$ direction, while the $u$ value of curves $c_{1}, c_{2}, c_{3}$, and blade tip are $u_{1}, u_{2}, u_{3}$, and 1 , respectively, as shown in Fig. 6 . To guarantee the uniformity of allowance on the machined surface, the allowance in finish milling region is linearly interpolated as follows. 


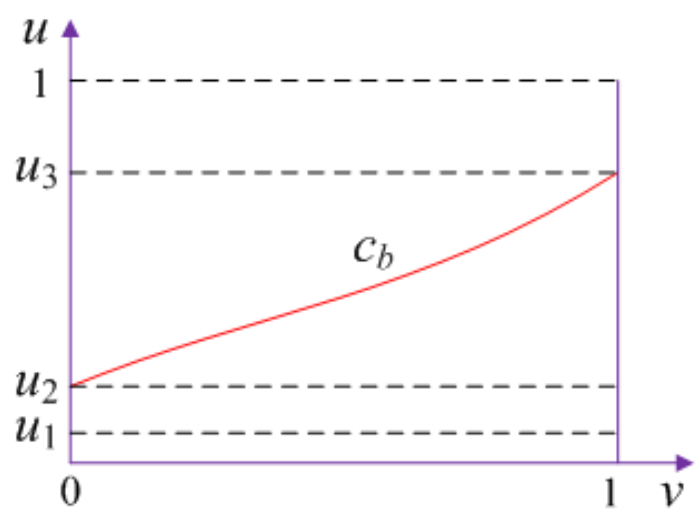

Fig. 6 Curve parameters in $u$ direction

Step 1 Suppose curve $c_{b}$ can be expressed as

$$
v=f(u), u \in\left(u_{2}, u_{3}\right)
$$

Boundary conditions of allowance distribution are listed as

$$
\begin{aligned}
& \Delta\left(u_{1}, 0\right)=\Delta \max \\
& \Delta\left(u_{1}, 1\right)=\Delta \max \\
& \left.\Delta(u, v)\right|_{v=f(u), u \in\left(u_{2}, u_{3}\right)}=\Delta \min
\end{aligned}
$$

Step 2 Calculate the allowance on the left and right boundaries of this region by interpolating along the $u$ direction.

$$
\begin{array}{ll}
\Delta(u, 0)=\Delta \max -\frac{\Delta \max -\Delta \min }{u_{2}-u_{1}} \cdot u, u \in\left(u_{1}, u_{2}\right) \\
\Delta(u, 1)=\Delta \max -\frac{\Delta \max -\Delta \min }{u_{3}-u_{1}} \cdot u, u \in\left(u_{2}, u_{3}\right)
\end{array}
$$

Step 3 Calculate the allowance at each CL points by interpolating along the $v$ direction. When $u \in\left[u_{1}, u_{2}\right)$, the allowance is calculated as shown in Eq. 10 .

$$
\Delta(u, v)=\Delta(u, 0)+\frac{\Delta(u, 1)-\Delta(u, 0)}{1-0} \cdot v
$$

Submit Eqs. 8 and 9 into Eq. 10, the allowance can be written as

$$
\Delta(u, v)=\Delta \max +\left(\frac{v-1}{u_{2}-u_{1}}-\frac{v}{u_{3}-u_{1}}\right)(\Delta \max -\Delta \min ) \cdot u
$$

When $u \in\left[u_{2}, u_{3}\right]$ and $v>f(u)$, the allowance is calculated as

$$
\Delta(u, v)=\Delta \min +\frac{\Delta(u, 1)-\Delta \min }{1-f(u)} \cdot v
$$

When $u \in\left[u_{2}, u_{3}\right]$ and $v \leq f(u)$, the allowance is the same as region $S_{3}^{\prime}$, as shown in Eq. 13 . 


$$
\Delta(u, v)=\Delta \min
$$

When $u \in\left(u_{3}, 1\right]$, this region is the completely repaired region $S_{3}^{\prime}$. The allowance can also be presented with Eq. 13.

\section{Conclusions}

A tool path planning approach is proposed in this paper to machine repaired blades of blisks in rough and finish milling. The main contributions of this work include that (a) allowance-based region dividing methods for machining repaired blades, (b) an iso-stepover method to plan tool path in rough milling, and (c) an adaptive tool path planning approach based on non-uniform allowance distributions. This work can be used to plan tool path in milling of other repaired parts with irregular allowance.

\section{References}

[1] GAO J, CHEN X, YILMAZ O, et al. An integrated adaptive repair solution for complex aerospace components through geometry reconstruction[J]. International Journal of Advanced Manufacturing Technology, 2008, 36(11-12):1170-1179

[2] BREMER C. Adaptive machining technology and data management for automated repair of complex turbine components with focus on blisk repair. http://www.bct-online.de

[3] YILMAZ ONOBLE D, GINDY N N Z, et al. A study of turbomachinery components machining and repairing methodologies[J]. Aircraft Engineering and Aerospace Technology. An International Journal, 2005, 77(6): 455-466

[4] Xue Y H, Zhao J P, Jing Z G, et al. Point cloud data registration and surface subdivision technology[M]. Beijin. National Defence Industry Press, 2011.(in Chinese)

[5] Yang D W. Open blisk machining based on the control curve[D]. Xi'an: Northwestern Polytechnical University, 2012.3, (in Chinese)

[6] Wu L P, Shi F Z, Chen W D,. Interference-free cutter location data computation for flat-endmill in 4-Axis NC machining[J]. Journal of Beijing University of Aeronautics and Astronautics, 2002, 28(6): 625-628, (in Chinese)

[7] Ren J X, Yang D W, Yao C F, et al. A cutter-axis vector control method for open blisk 4-axis NC machining based on the control curve. Acta Aeronautica et Astronautica Sinica, 2012. 33(8): 1515-1523, (in Chinese)

This work is supported by the national science and technology major projects [grant number 2013ZX04011031]. 